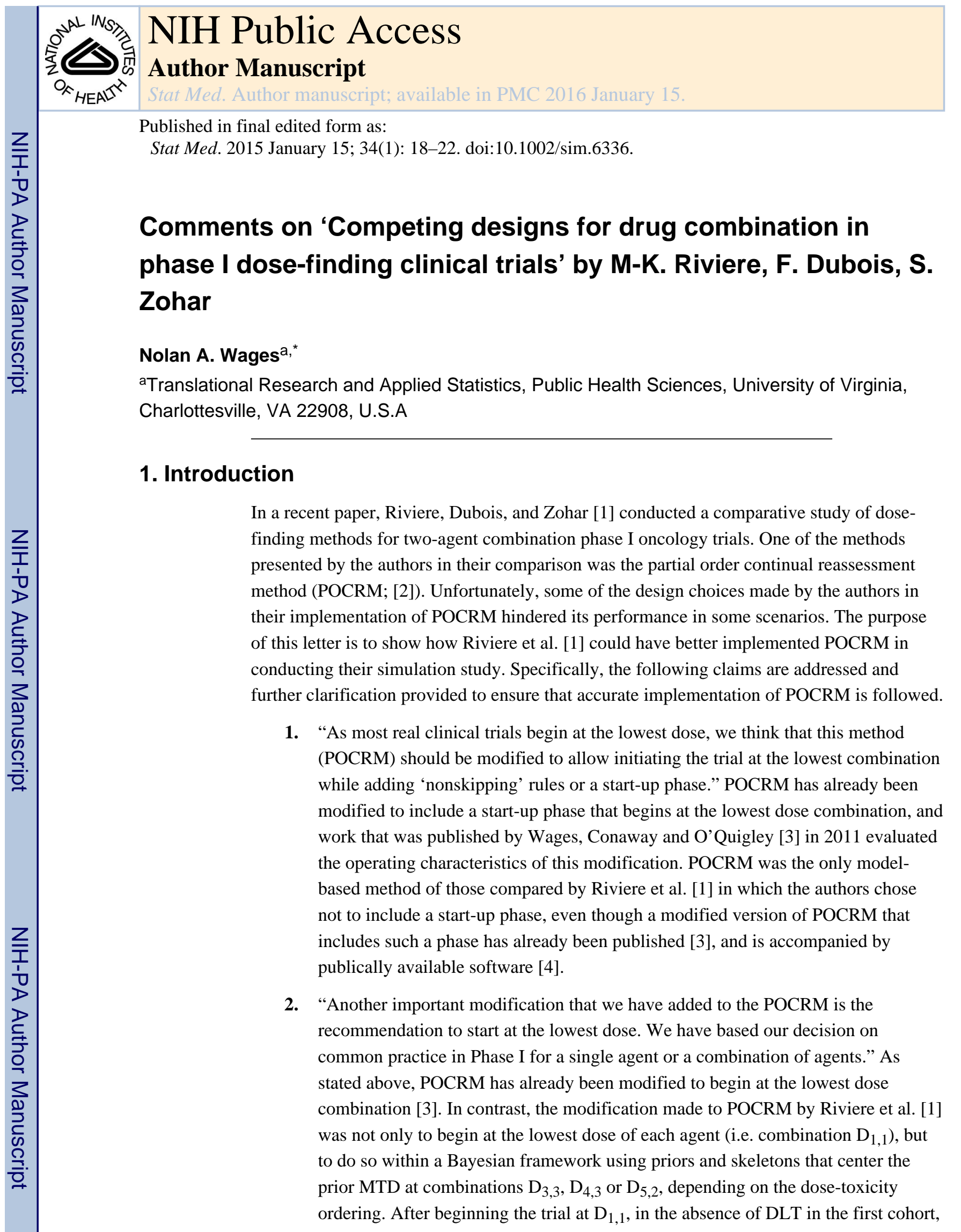

*Correspondence to: Nolan A. Wages, Translational Research and Applied Statistics, Public Health Sciences, University of Virginia, Charlottesville, VA 22908, U.S.A. nwages@ virginia.edu. 
this modification will cause the design to gravitate toward the prior MTD (i.e. $\mathrm{D}_{3,3}$, $\mathrm{D}_{4,3}$ or $\mathrm{D}_{5,2}$ ), and such behavior would not be allowed in practice, as pointed out by the authors. Therefore, the modification made by the authors does not resolve any practical issue associated with the POCRM. The authors have chosen to make a modification that is not allowable in practice within an article that is meant to inform statisticians about how best to practically conduct Phase I trials of combinations.

Wages and Conaway [5] published practical guidelines for implementing POCRM, yet the comparison by Riviere et al. [1] did not follow these recommendations, especially in choosing an appropriate subset of possible dose-toxicity orderings. The guidelines in [5], combined with an $\mathbf{R}$ package (pocrm [4]), have led to the successful implementation of POCRM in two ongoing trials since its publication in 2011. One recently completed accrual and is in follow-up (Mel 58) and the other (Mel 60) is open to accrual at two sites, the University of Virginia Cancer Center and MD Anderson Cancer Center. Descriptions of these trials can be found at https://clinicaltrials.gov/ct2/show/NCT01585350? term $=$ mel58\&rank=1 and https://clinicaltrials.gov/ct2/show/NCT02126579? term=mel60\&rank=1. The use of appropriate POCRM specifications in the comparative work of Riviere et al. [1] would have resulted in a more accurate evaluation of POCRM's design characteristics, and provided the readers of Statistics in Medicine valuable insight on how the method fares relative to existing methods. In this letter, we focus our attention on proper selection of possible dose-toxicity orderings for use in the POCRM. In the subsequent sections, we review the recommendations of Wages and Conaway [5] in choosing orderings, and discuss their impact on the operating characteristics of POCRM. This sheds a very different light on the results and conclusions of Riviere [1].

\section{A recommended subset of orderings}

Wages and Conaway [5] provide a discussion of the problem of partial ordering in dosefinding in a matrix of drug combinations. Because the dimension of a drug combination matrix can be quite large, it is often not feasible to enumerate all possible dose-toxicity orderings of the combinations. The primary objective in Wages and Conaway [5] was to provide guidance in choosing a proper subset of possible dose-toxicity orderings, according to the known ordering information among combinations, for use in the POCRM. Based on simulation studies in Wages and Conaway [5], the following recommendations were made:

1. Choose a subset of approximately $6-9$ orderings. This provides an appropriate balance between choosing enough orderings so that we include adequate information to account for the uncertainty surrounding partially ordered dosetoxicity curves, without increasing the dimension of the problem so much so that we diminish performance. Simulation results in [5] show that using three orderings is not sufficient in some scenarios, which was an important modification made to the original work by Wages et al. [2].

2. Arrange orderings according to movements across rows, up columns and along diagonals. Since, in a large matrix, there could many ways to arrange combinations 
along a diagonal, restrict movements to only moving across rows, up columns, and up or down any diagonal.

The POCRM can certainly accommodate other subset sizes consisting of alternative orderings, should we have some ordering information at our disposal. If, however, the only information we have is the assumption of a monotonicity across rows and up columns of the matrix, then we can use the guidelines of Wages and Conaway [5] that form a "default" subset of six orderings and have confidence in its performance, as indicated in the simulation results below. Following Riviere et al. [1], suppose the drug combinations are denoted $D_{j, k}$, where $j=1, \ldots, J$ refers to the level of agent 1 , and $k=1, \ldots, K$ refers to the level of agent 2. The simulation results evaluate the performance of methods for $J=5$ levels of agent 1 , and $K=3$ levels of agent 2, for a total of 15 combinations. The guidelines of Wages and Conaway [5] recommend the following subset of six orderings, according to the combination labels in Figure 1 of Riviere [1].

1. Across rows

$$
\begin{aligned}
& D_{1,1} \rightarrow D_{2,1} \rightarrow D_{3,1} \rightarrow D_{4,1} \rightarrow D_{5,1} \rightarrow D_{1,2} \rightarrow D_{2,2} \rightarrow D_{3,2} \rightarrow D_{4,2} \rightarrow D_{5,2} \rightarrow D_{1,3} \\
& \rightarrow D_{2,3} \rightarrow D_{3,3} \rightarrow D_{4,3} \rightarrow D_{5,3}
\end{aligned}
$$

2. Up columns

$$
\begin{aligned}
& D_{1,1} \rightarrow D_{1,2} \rightarrow D_{1,3} \rightarrow D_{2,1} \rightarrow D_{2,2} \rightarrow D_{2,3} \rightarrow D_{3,1} \rightarrow D_{3,2} \rightarrow D_{3,3} \rightarrow D_{4,1} \rightarrow D_{4,2} \\
& \rightarrow D_{4,3} \rightarrow D_{5,1} \rightarrow D_{5,2} \rightarrow D_{5,3}
\end{aligned}
$$

3. Up diagonals

$$
\begin{aligned}
& D_{1,1} \rightarrow D_{2,1} \rightarrow D_{1,2} \rightarrow D_{3,1} \rightarrow D_{2,2} \rightarrow D_{1,3} \rightarrow D_{4,1} \rightarrow D_{3,2} \rightarrow D_{2,3} \rightarrow D_{5,1} \rightarrow D_{4,2} \\
& \rightarrow D_{3,3} \rightarrow D_{5,2} \rightarrow D_{4,3} \rightarrow D_{5,3}
\end{aligned}
$$

4. Down diagonals

$$
\begin{aligned}
& D_{1,1} \rightarrow D_{1,2} \rightarrow D_{2,1} \rightarrow D_{1,3} \rightarrow D_{2,2} \rightarrow D_{3,1} \rightarrow D_{2,3} \rightarrow D_{3,2} \rightarrow D_{4,1} \rightarrow D_{3,3} \rightarrow D_{4,2} \\
& \rightarrow D_{5,1} \rightarrow D_{4,3} \rightarrow D_{5,2} \rightarrow D_{5,3}
\end{aligned}
$$

5. Alternating down-up diagonals

$$
\begin{aligned}
& D_{1,1} \rightarrow D_{2,1} \rightarrow D_{1,2} \rightarrow D_{1,3} \rightarrow D_{2,2} \rightarrow D_{3,1} \rightarrow D_{4,1} \rightarrow D_{3,2} \rightarrow D_{2,3} \rightarrow D_{3,3} \rightarrow D_{4,2} \\
& \rightarrow D_{5,1} \rightarrow D_{5,2} \rightarrow D_{4,3} \rightarrow D_{5,3}
\end{aligned}
$$

6. Alternating up-down diagonals

$$
\begin{aligned}
& D_{1,1} \rightarrow D_{1,2} \rightarrow D_{2,1} \rightarrow D_{3,1} \rightarrow D_{2,2} \rightarrow D_{1,3} \rightarrow D_{2,3} \rightarrow D_{3,2} \rightarrow D_{4,1} \rightarrow D_{5,1} \rightarrow D_{4,2} \\
& \rightarrow D_{3,3} \rightarrow D_{4,3} \rightarrow D_{5,2} \rightarrow D_{5,3}
\end{aligned}
$$

Riviere et al. [1] uses a subset of three orderings that includes (1) alternating down-up diagonals, (2) down diagonals, and (3) $D_{1,1} \rightarrow D_{2,1} \rightarrow D_{3,1} \rightarrow D_{1,2} \rightarrow D_{2,2} \rightarrow D_{1,3} \rightarrow D_{2,3}$ $\rightarrow D_{4,1} \rightarrow D_{5,1} \rightarrow D_{3,2} \rightarrow D_{4,2} \rightarrow D_{5,2} \rightarrow D_{3,3} \rightarrow D_{4,3} \rightarrow D_{5,3}$. The authors do not provide any justification for choosing ordering (3). The lack of sufficient ordering information contained in the subset used by Riviere et al. [1] significantly diminishes the performance of POCRM in some scenarios ( $\mathrm{Sc} 9, \mathrm{Sc} 10)$, when compared to using recommended specifications [5] (see simulation results below). The Supplemental Material (Section 1.2.2) of Riviere et al. [1] evaluates the influence of the number of orderings on the POCRM, yet it is not clear 
which orderings were used in this assessment beyond the three implemented in the paper. The study of orderings conducted by the authors in the Supplemental Material should at least have included the "default" subset above recommended by Wages and Conaway [5]. Using this recommended subset, we re-simulate the POCRM, and examine its operating characteristics.

\section{Simulation studies using POCRM guidelines}

We carried out simulation studies on the 10 true toxicity scenarios in Riviere [1]. For each scenario, 2000 simulated trials were run and each trial exhausted a sample size of $n=60$ patients.

\subsection{Bayesian POCRM using recommended orderings}

Riviere et al. [1] presents and compares the Bayesian form of the POCRM [2], after making the modification of beginning the trial at $D_{1,1}$, as discussed above. In the first set of simulations, we are interested in evaluating the operating characteristics of Bayesian POCRM using the recommended guidelines of Wages and Conaway [5] for specifying a subset of orderings. The other design configurations for POCRM are the same as those specified by Riviere et al. [1]. The skeleton values used in simulation were $\{0.0001,0.0006$, $0.002,0.005,0.01,0.02,0.04,0.06,0.10,0.14,0.19,0.24,0.30,0.36,0.42\}$, termed WMP4

[1]. The location of these skeleton values were adjusted in order to correspond to six possible orderings of DLT probabilities above using the getwm function in $\mathbf{R}$ package pocrm [4]. The cohort size was set to 3 throughout each trial. These Bayesian results are included solely for the purpose of comparing performance of POCRM using an appropriate subset of orderings to that of POCRM using the choices made by Riviere et al. [1]. As noted, the difference between the prior specifications (prior distribution, skeletons, and orderings) and the starting combination create a possible escalation after the first cohort of patients (if no DLT) that would not be allowed in practice. As discussed below, the impact of this choice is most evident in the results for number of DLT's induced in Table 1. In practice, we recommend the following two-stage design of Wages et al. [3, 5].

\subsection{Two-stage POCRM using recommended orderings}

In the second set of simulation results, we take the approach of beginning at $D_{1,1}$ and using a start-up phase to slowly escalate through the combinations before the first observed DLT, before basing allocation on the model-recommended combination. This is the approach implemented in the trials referenced in Section 1. We make use of "zoning" the combinations according to the diagonals of the matrix. The trial begins in zone $Z_{1}=\left\{D_{1,1}\right\}$ and the first cohort of patients is enrolled on the lowest combination. As long as no DLT's occur, cohorts of patients are examined at each combination within the currently occupied zone, before escalating to the next highest zone. If $D_{11}$ was tried and deemed "safe", the trial escalates to zone $Z_{2}=\left\{D_{1,2}, D_{2,1}\right\}$. The trial is not allowed to advance to zone $Z_{3}=\left\{D_{1,3}\right.$, $\left.D_{2,2}, D_{3,1}\right\}$ in the start-up phase until a cohort of patients has been observed at all combinations in $Z_{2}$. In both trials mentioned above, escalation in the start-up phase occurs in cohorts of 2, and continues until the first DLT is observed. Subsequent to a DLT being observed, the modeling phase of the trial begins and accrues eligible patients in cohorts of 
size 1. All simulations were carried out using the pocrm.sim function of $\mathbf{R}$ package pocrm [4].

\subsection{Results and conclusions}

Table 1 reports simulation results using the choices of Riviere et al. [1], as well as using the ordering guidelines of Wages and Conaway [5] for both the Bayesian and likelihood form of POCRM. Several conclusions reached through examination of the results in Table 1 are in stark contrast to those reached by Riviere et al. [1].

1. "In Scenarios 9 and $10, \ldots$, the algorithm-based methods performed better than the model-based methods." It is clear from Table 1 that, when using orderings 1-6 above, the PCS for POCRM increased from 3.4\% to 53.4\% in Scenario 9, and from $8.2 \%$ to $50.0 \%$ in Scenario 10. In Scenario 9, when looking at the best performing algorithm-based design (AISO), the PCS for POCRM is $28.6 \%$ higher (53.4\% vs. 24.8\%). In Scenario 10, when looking at the best performing algorithm-based design (AISO), the PCS for POCRM is $18.2 \%$ higher (50.0\% vs. $31.8 \%$ ). Not only are the POCRM percentages substantially higher than their algorithm-based counterparts, but they are also much higher than any other model-based method (see Table II [1]). In Scenario 9, the 53.4\% PCS for POCRM is $47.4 \%$ higher than the best performing model-based design (6\% for BGUMBEL). In Scenario 10, the $51.2 \%$ for POCRM is $33.7 \%$ higher than the best performing model-based design (16.3\% for BCOPULA). Similar conclusions can be made with regards to the twostage design.

2. "Table II shows that POCRM generated more DLT's than the other methods." This was true in [1] but is a result of POCRM being the only method evaluated in which a start-up phase was not implemented by the authors. Using a the start-up phase, Table 1 indicates that the mean number of DLT's for POCRM decreased from 19.7 to 16.6 , which corresponds to an overall DLT rate (27.7\%) that is very close to, but slightly below, the target DLT rate of $30 \%$. When using a start-up phase, the POCRM no longer has the highest number of DLT's generated on average (TSTAT; 17.3).

3. "In our comparative study, none of the model-based designs gave consistently better results than the others." It is unexpected that a particular method will outperform five competing methods in each of 10 scenarios by a substantial margin. However, if the new POCRM results in Table 1 were used in place of those in Riviere et al. [1], it could be seen that POCRM yields the largest PCS of the multi-dimensional methods in 5 of 10 scenarios (Sc1, Sc6, Sc8, Sc9, Sc10), and in only one of those scenarios ( $\mathrm{Sc} 1$ ) was the gain less than $4 \%$ over the next highest PCS. In Scenario 3, the Bayesian POCRM results in the largest PCS, while the PCS of two-stage POCRM is just below that of BCOPULA. POCRM is still performing well in the other four scenarios, essentially tying BCOPULA for the highest PCS in Sc7. Of course, there will always be certain scenarios in which some methods perform better than others. Therefore, a useful tool in comparing dose-finding designs can be average performance for all scenarios, as mentioned by the authors 
in Section 5 in reference to their choice of skeleton. When using appropriate specifications, the average PCS across the 10 scenarios is $61.45 \%$ for Bayesian POCRM (63.22\% for MLE) and 52.96\% for the next highest performing multidimensional method (I2D), a difference of $\approx 10 \%$. The notion of average performance is especially critical when evaluating the 'CRM anti-diag' designs. These designs yield the largest PCS values in the first 8 scenarios, which should be expected since they implement CRM on a completely ordered set of seven combinations, rather than on a partially ordered set of fifteen. However, if the true MTD is not captured in the chosen subset, then the PCS will be 0\%, as in Scenarios 9 and 10. Although, the PCS is highest in Scenarios 1-8, the 0's in Scenarios 9 and 10 drop the overall average PCS of these designs to $61.4 \%$ and $62.1 \%$, respectively. Although these percentages are close to the average PCS for POCRM, investigators do not run the risk of missing the MTD combination altogether, thus obtaining PCS $0 \%$, when using POCRM.

Based on these new results, we come to quite a different conclusion than Riviere et al. [1] regarding the comparative performance of the competing methods.

\section{Acknowledgments}

The project was supported by Grant Number 1K25CA181638-01A1 from the National Cancer Institute.

\section{References}

1. Riviere MK, Dubois F, Zohar S. Competing designs for drug combination in phase I dose-finding clinical trials. Statistics in Medicine. 201410.1002/sim.6094

2. Wages NA, Conaway MR, O'Quigley J. Continual reassessment method for partial ordering. Biometrics. 2011; 67:1555-63. [PubMed: 21361888]

3. Wages NA, Conaway MR, O'Quigley J. Dose finding design for multi-drug combinations. Clinical Trials. 2011; 8:380-389. [PubMed: 21652689]

4. Wages NA, Varhegyi N. pocrm: an R package for phase I trials of combinations of agents. Computer Methods \& Programs in Biomedicine. 2013; 112:211-218. [PubMed: 23871691]

5. Wages NA, Conaway MR. Specifications of a continual reassessment method design for phase I trials of combined drugs. Pharmaceutical Statistics. 2013; 12:217-224. [PubMed: 23729323] 


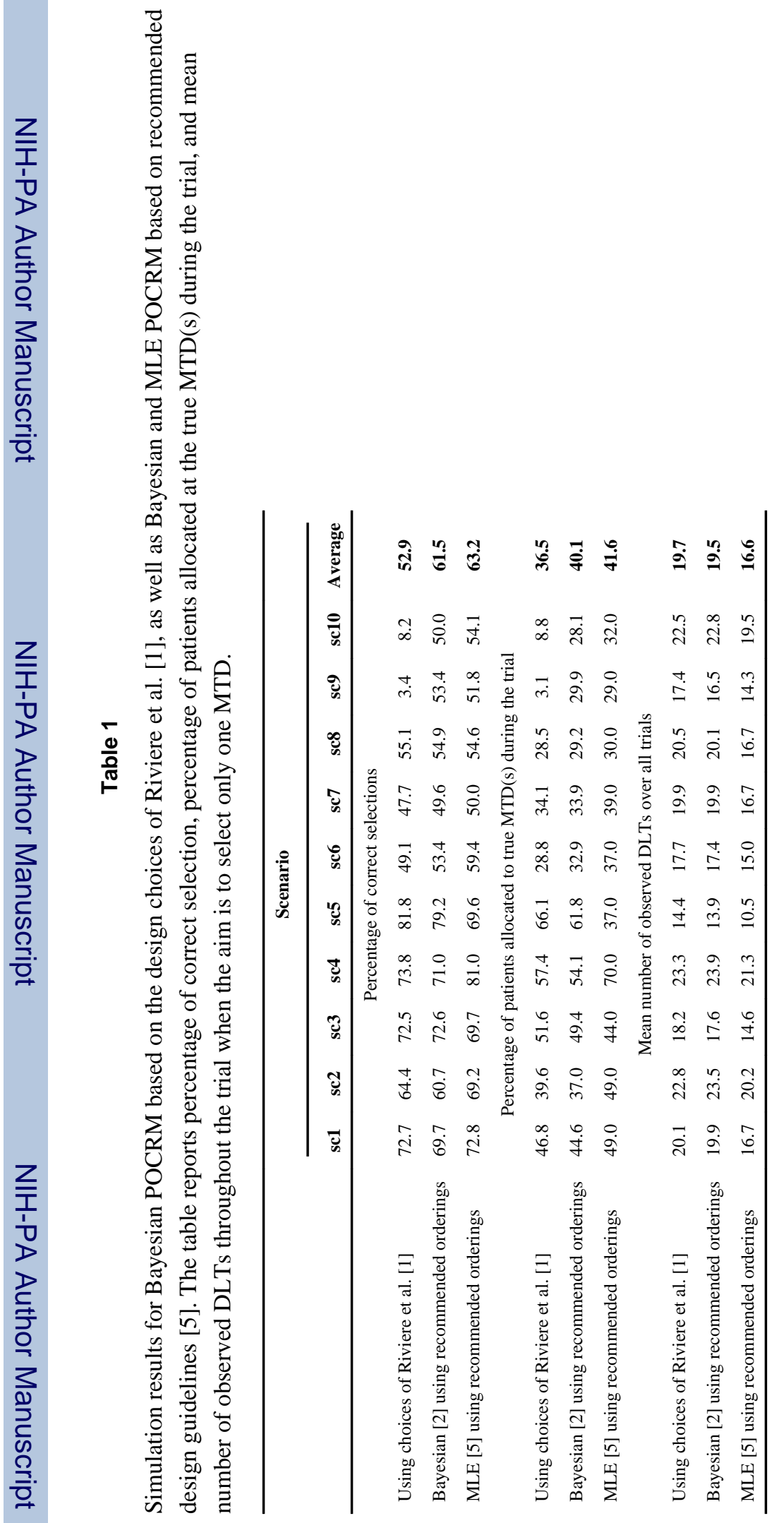

